\title{
Sharp Differentiability Results for the Lower Local Lipschitz Constant and Applications to Non-embedding
}

\author{
K. Wildrick • T. Zürcher
}

Received: 10 September 2013 / Published online: 13 August 2014

(C) Mathematica Josephina, Inc. 2014

\begin{abstract}
We give a sharp condition on the lower local Lipschitz constant of a mapping from a metric space supporting a Poincaré inequality to a Banach space with the Radon-Nikodym property that guarantees differentiability at almost every point. We apply these results to obtain a non-embedding theorem for a corresponding class of mappings.
\end{abstract}

Keywords Differentiability $\cdot$ Poincaré inequality $\cdot$ Lorentz space $\cdot$ Sobolev space . Embedding

Mathematics Subject Classification $26 \mathrm{~B} 05 \cdot 30 \mathrm{~L} 05$

\section{Introduction}

In 1919, Rademacher proved that Lipschitz mappings between Euclidean spaces are differentiable almost everywhere [33]. As the Lipschitz condition is global, while differentiability is local, Stepanov considered the set

Communicated by Loukas Grafakos.

\footnotetext{
K. Wildrick $(\bowtie)$

Département de mathématiques, Université de Fribourg, Chemin du Musée 23, 1700 Fribourg, Switzerland e-mail: kevin.wildrick@unifr.ch; quasikevin@gmail.com

T. Zürcher

Jyväskylän yliopisto, Matematiikan ja tilastotieteen laitos, PL 35 (MaD), 40014 Jyväskylä, Finland

e-mail: thomas.t.zurcher@jyu.fi 


$$
S(f):=\left\{x \in \mathbb{R}^{n}: \operatorname{Lip} f(x)<\infty\right\},
$$

where

$$
\operatorname{Lip} f(x):=\limsup _{r \rightarrow 0} \frac{\sup _{y \in B(x, r)}|f(y)-f(x)|}{r} .
$$

His arguments resulted in the following generalization of Rademacher's theorem: A function $f: \mathbb{R}^{n} \rightarrow \mathbb{R}$ is differentiable almost everywhere in $S(f)$ [38]. A second strengthening of Rademacher's theorem states that if $p>n$, then Sobolev functions in $W_{\text {loc }}^{1, p}\left(\mathbb{R}^{n}\right)$ have representatives that are differentiable almost everywhere; this is due to Cesari [6] when $n=2$. Calderón [5] and later Stein [39] generalized and sharpened this result: If the weak gradient of a function on $\mathbb{R}^{n}$ is in the Lorentz space $L^{n, 1}$, then the function has a continuous representative that is differentiable almost everywhere. Moreover, $L^{n, 1}$ is the largest of the Lorentz spaces $\left\{L^{n, q}: 1 \leq\right.$ $q \leq n\}$ to have this property. In fact, Calderón considered Orlicz spaces; the relation between these spaces and the Lorentz spaces is clarified in [23] and [31]. Heuristically, a function on $\mathbb{R}^{n}$ with a weak gradient in $L^{n, 1}$ shares properties with absolutely continuous functions of a single variable. This principle has been extended to include various higher-dimensional notions of absolute continuity [23,29], and to apply in more general settings [34,35,41].

Yet another generalization of Rademacher's theorem was explored by Balogh and Csörnyei, who considered the lower local Lipschitz constant

$$
\operatorname{lip} f(x):=\liminf _{r \rightarrow 0} \frac{\sup _{y \in B(x, r)}|f(y)-f(x)|}{r}
$$

instead of Lip $f$ [2]. They observed that Stepanov's theorem with Lip $f$ replaced by lip $f$ does not hold in general, and provided two examples highlighting the obstructions. The first showed that control on the integrability of lip $f$ is needed, and the second showed that an upper bound on the size of the set where lip $f$ is infinite is required. On the other hand, they gave the following positive result, which, when combined with the $n$-dimensional version of Cesari's differentiability result, can be considered to be of "Stepanov-type".

Theorem 1.1 (Balogh-Csörnyei) Let $\Omega \subseteq \mathbb{R}^{n}$ be a domain, and let $f: \Omega \rightarrow \mathbb{R}$ be a continuous function. Assume that $\operatorname{lip} f(x)<\infty$ for $x \in \Omega \backslash E$, where the set $E$ has $\sigma$-finite $(n-1)$-dimensional Hausdorff measure, and that lip $f \in L_{\mathrm{loc}}^{p}(\Omega)$ for some $1 \leq p \leq \infty$. Then $f \in W_{\mathrm{loc}}^{1, p}(\Omega)$.

In the foundational work [7], Cheeger generalized Rademacher's theorem to the setting of Lipschitz functions on metric measure spaces that support a Poincaré inequality using the notion of a measurable differentiable structure. Important work of Keith [22] and other generalizations in this setting followed $[3,9,42]$.

The first result of this paper sharpens the theorem of Balogh and Csörnyei to the Lorentz scale, in the spirit of Stein. Our result is in the setting of mappings between metric measure spaces, although it is new even for functions on Euclidean space. The assumptions are standard for this setting, and can be heuristically understood to 
mean that the domain space $(X, d, \mu)$ is of dimension $Q$ and contains a large-enough collection of rectifiable curves to support "first-order calculus". For more details on these definitions, as well as the usual generalization of Lip and lip to the setting of mappings between metric spaces, see Sect. 2 below.

Theorem 1.2 Let $Q \geq 1$ and $1 \leq q \leq Q$. Let $(X, d, \mu)$ be a complete and Ahlfors $Q$-regular metric space that supports a $q$-Poincaré inequality, and let $Y$ be any metric space. Consider a continuous mapping $f: X \rightarrow Y$, and set

$$
E=\{x \in X: \operatorname{lip} f(x)=\infty\}
$$

If $\operatorname{lip} f \in L^{Q, 1}(X)$ and either

- $q=1$ and $E$ has $\sigma$ finite $(Q-1)$-dimensional Hausdorff measure, or

- $q>1$ and $E$ has Hausdorff dimension at most $(Q-q)$,

then $f$ has an upper gradient in the Lorentz space $L^{Q, 1}(X)$.

We note that a similar result at the Lebesgue scale for real-valued functions was proven by the second author in [42]. The main additional ingredient needed for the proof of Theorem 1.2 is an interpolation result that implies the boundedness of a suitable maximal function operator on the relevant Lorentz space.

As in the Euclidean setting, the assumption that an upper gradient of the mapping $f$ is in the Lorentz space $L^{Q, 1}(X)$ implies that $f$ shares many properties with an absolutely continuous function on $\mathbb{R}$; see Theorem 6.3, Proposition 6.4, Corollary 6.7, and Lemma 6.8 in [41]. We record some of these properties in the following statement.

Corollary 1.3 Assume the notation and hypotheses of Theorem 1.2. If $N \subseteq X$ is a set of zero $Q$-dimensional Hausdorff measure, then so is $f(N)$. Moreover, the image $f(X)$ is the countable union of Lipschitz images of $X$ and a set of zero $Q$ dimensional Hausdorff measure. In particular, if $X=\mathbb{R}^{n}$, then $f(X)$ is a countably $n$-rectifiable subset of $Y$.

Hanson has recently given an example showing that Theorem 1.2 is quite sharp with respect to the size of the exceptional set [14, Theorem 2.3]. Here we provide an example that shows sharpness with respect to the integrability of lip. Our construction draws on ideas of Hanson, Malý [30], and our previous work on the capacity of points [41]. It is substantially different from the corresponding example in [2].

Theorem 1.4 Let $n \geq 2$ be an integer, and let $\mathcal{S}$ be a rearrangement invariant Banach function space containing a compactly supported function $g \notin L^{n, 1}\left(\mathbb{R}^{n}\right)$. Then there exists a compactly supported continuous function $f: \mathbb{R}^{n} \rightarrow \mathbb{R}$ with the following properties:

- $\operatorname{lip} f(x)<\infty$ for all $x \in \mathbb{R}^{n}$,

- $\operatorname{lip} f(x) \in \mathcal{S}$,

- the set of points at which $f$ fails to be differentiable has positive $n$-dimensional Hausdorff measure. 
A reader unfamiliar with the notion of a rearrangement invariant Banach function space may consult [4]. As an example, we point out that for any $1<q \leq n$, the Lorentz space $L^{n, q}\left(\mathbb{R}^{n}\right)$ satisfies the hypotheses of the above theorem.

In the case that the target of the mapping in Theorem 1.2 is a Banach space with the Radon-Nikodym property, in Theorem 4.2 below we provide a differentiation result in the spirit of Stein. Here differentiability is meant in the sense of Cheeger and Kleiner [7,9], i.e., in terms of a measurable differentiable structure. See Sect. 2.3 below for more details and definitions.

Theorem 1.5 Let $Q \geq 1$. Let $(X, d, \mu)$ be a complete and Ahlfors $Q$-regular metric space that supports a $Q$-Poincaré inequality, and let $V$ be a Banach space with the Radon-Nikodym property. Let $f: X \rightarrow V$ be a continuous mapping with an upper gradient in the Lorentz space $L^{Q, 1}(X)$. Then $f$ is differentiable almost everywhere with respect to any measurable differentiable structure on $X$.

Theorem 1.5, when combined with Theorem 1.2, provides a "Stepanov-Stein" result for lip.

Expanding on Keith's results of [22], recent work of Gong [13] has further clarified the connection between the existence of a measurable differentiable structure and the relationship between Lip and lip for Lipschitz functions (see also [36]). In particular, it follows from [13] that a complete and doubling metric measure space $X$ supports a measurable differentiable structure if and only if $X$ can be countably partitioned, up to a null set, into sets on which Lip and lip are uniformly comparable for all Lipschitz functions. It would be interesting to know if this comparability extends to mappings with an upper gradient in the Lorentz space $L^{Q, 1}$. Such comparability need not be true even for mappings with the property that lip vanishes almost everywhere, as shown by Balogh and Csörnyei [2, Theorem 1.4].

As discussed in [9], differentiability theorems in the setting of metric measure spaces lead to corresponding non-embedding theorems. In certain cases, these nonembedding results have important applications in theoretical computer science [10].

The differentiability of Lipschitz mappings into a Banach space with the RadonNikodym property leads to a bi-Lipschitz non-embedding theorem. Our differentiation result Theorem 1.5 leads to a non-embedding theorem for a larger class of mappings.

For a mapping $f: X \rightarrow Y$ of metric spaces, we denote

$$
\mathcal{S}_{f}=\{x \in X: \operatorname{Lip} f(x)<\infty\} .
$$

We say that $f$ is an embedding if it is a homeomorphism of $X$ onto $f(X)$.

Theorem 1.6 Let $Q \geq 1$. Let $(X, d, \mu)$ be a complete and Ahlfors $Q$-regular metric space that supports a $Q$-Poincaré inequality, and let $V$ be a Banach space with the Radon-Nikodym property. Let $\iota: X \rightarrow V$ be an embedding with an upper gradient in the Lorentz space $L^{Q, 1}(X)$. If

(i) $\mathcal{H}_{V}^{Q}\left(\iota(X) \backslash \mathcal{S}_{\iota-1}\right)=0$,

(ii) $\mathcal{H}_{X}^{Q}\left(X \backslash \iota^{-1}\left(\mathcal{S}_{\iota-1}\right)\right)=0$,

then for any coordinate chart $\left(X_{\alpha}, \varphi_{\alpha}\right)$ of any measurable differentiable structure on $X$, it holds that $Q \leq N(\alpha)$. 
Combining Theorem 1.2 with Theorem 1.6 produces a non-embedding result for lip.

We note that in the setting of Theorem 1.6, the existence of an upper gradient of $\iota$ in $L^{Q, 1}(X)$ implies that Lip $\iota(x)<\infty$ at $\mathcal{H}^{Q}$-almost every point, and moreover it implies that $\iota$ maps $\mathcal{H}_{X}^{Q}$-null sets to $\mathcal{H}_{V}^{Q}$-null sets [41, Sect. 6]. Hence, if it is also known that the image of $\iota$ is an Ahlfors $Q$-regular metric space supporting a $Q$-Poincaré inequality, then conditions (i) and (ii) above could be replaced by the symmetric requirement that $\iota^{-1}$ have an upper gradient in $L^{Q, 1}(\iota(X))$.

Let us consider the concrete example of the Heisenberg group $\mathbb{H}$, which is an Ahlfors 4-regular metric measure space, is homeomorphic to $\mathbb{R}^{3}$, supports a 1 Poincaré inequality, and has a measurable differentiable structure in which each chart has dimension $2[19,24,32]$. Hence, in the language of Theorem 1.6, $Q>N(\alpha)$ for each $\alpha$. The differentiability theorem for Lipschitz mappings into a Banach space $V$ with the Radon-Nikodym property given in [9] allows one to prove that there is no bi-Lipschitz embedding $\iota: \mathbb{H} \rightarrow V$. Our result states that there is not even an embedding $\iota: \mathbb{H} \rightarrow V$ satisfying the hypotheses of Theorem 1.6, which are much weaker than the bi-Lipschitz condition. We point out that the identity mapping from $\mathbb{H}$ to $\mathbb{R}^{3}$ is Lipschitz on compact sets, and so some metric condition on the inverse of the embedding is needed.

The standard assumptions of Ahlfors regularity and a Poincaré inequality in the above theorems place conditions on large scales that are not natural for the conclusions, which are local in nature. However, the theorems still hold for arbitrary domains in Euclidean space, as can be seen by restriction to a sufficiently small closed ball. Moreover, it appears that Theorems 1.2, 1.5, and 1.6 remain valid when small scale and localized versions of these assumptions, as well as a local Lorentz integrability condition, are used instead. We have used global assumptions only for clarity of exposition, and we leave the aforementioned generalizations, which we expect can be proven in the same fashion, to the reader; see [41, Remark 6.11].

This paper is organized as follows. In Sect. 2 we establish notation and definitions regarding metric measure spaces, Lorentz integrability, and measurable differentiable structures. We then prove Theorem 1.2 in Sect. 3. This is followed in Sect. 4 by a proof of the differentiation result Theorem 1.5. Section 5 discusses the basic properties of weak tangents and weak tangent mappings at points of differentiability, which leads to a proof of the non-embedding result Theorem 1.6. Finally, we give the example described in Theorem 1.4 in Sect. 6.

We wish to thank Jeff Cheeger, Amiran Gogatishvili, Bruce Hanson, and Pekka Koskela for helpful comments. Further thanks goes to Mathematical Institute of the University of Bern, which was the affiliation of both authors at some point during the period of research for this paper.

\section{Notation and Basic Definitions}

\subsection{Metric Measure Spaces}

Given a metric space $(X, d)$, we denote the open ball centered at a point $x \in X$ of radius $r>0$ by

$$
B_{X}(x, r)=\{y \in X: d(x, y)<r\}
$$


and the corresponding closed ball by

$$
\bar{B}_{X}(x, r)=\{y \in X: d(x, y) \leq r\} .
$$

When there is no danger of confusion, we often write $B(x, r)$ in place of $B_{X}(x, r)$. A similar convention will be used for all objects that depend implicitly on the ambient space. Given a subset $A$ of $X$ and a number $\epsilon>0$, we notate the $\epsilon$-neighborhood of $A$ by

$$
\mathcal{N}(A, \epsilon)=\{x \in X: \operatorname{dist}(A, x)<\epsilon\} .
$$

Given an open ball $B=B(x, r)$ and a parameter $\lambda>0$, we set $\lambda B=B(x, \lambda r)$.

A metric measure space is a triple $(X, d, \mu)$ where $(X, d)$ is a metric space and $\mu$ is a measure on $X$. For our purposes, a measure is a nonnegative countably subadditive set function defined on all subsets of a measure space that gives the value 0 to the empty set. We further assume that measures are Borel inner and outer regular, and the collection of measurable sets is given by the completion of the Borel $\sigma$-algebra. We will often suppress the reference to the metric $d$ and the measure $\mu$ when they are understood.

The metric measure space $(X, d, \mu)$ is doubling if balls have finite and positive measure, and there is a constant $C \geq 1$ such $\mu(2 B) \leq C \mu(B)$ for any open ball $B$ in $X$. If $(X, d, \mu)$ is a doubling metric measure space, then the metric space $(X, d)$ enjoys the following property, also called doubling: there is a number $n \in \mathbb{N}$ such that any ball in $X$ of radius $r>0$ can be covered by at most $n$ balls of radius $r / 2$. It is easy to see that a doubling metric space is complete if and only if it is proper, i.e., closed and bounded sets are compact. Moreover, doubling metric spaces are separable.

The metric measure space $(X, d, \mu)$ is called Ahlfors $Q$-regular if there exists a constant $C \geq 1$ such that for each point $a \in X$ and each radius $0<r<2 \operatorname{diam} X$,

$$
\frac{r^{Q}}{C} \leq \mu(B(a, r)) \leq C r^{Q} .
$$

Note that an Ahlfors $Q$-regular space is doubling, quantitatively.

We denote the $Q$-dimensional Hausdorff measure on a metric space $(X, d)$ by $\mathcal{H}_{X}^{Q}$. If a metric measure space $(X, d, \mu)$ is Ahlfors $Q$-regular, then so is the metric measure space $\left(X, d, \mathcal{H}_{X}^{Q}\right)$, and so we will often work directly with the Hausdorff measure in this case.

\subsection{Upper Gradients and Poincaré Inequalities}

In this paper, the role of the norm of a gradient of a function on Euclidean space will be filled by the following notion more suited to the metric space setting. Let $f:\left(X, d_{X}, \mu\right) \rightarrow\left(Y, d_{Y}\right)$ be a mapping from a metric measure space to a metric space. An upper gradient of $f$ is a Borel function $g: X \rightarrow[0, \infty]$ such that for each rectifiable path $\gamma:[0,1] \rightarrow X$ 


$$
d_{Y}(f(\gamma(0)), f(\gamma(1))) \leq \int_{\gamma} g d s .
$$

Here and below $d s$ refers to integration with respect to arc-length. It is often difficult to verify equation (2) for every rectifiable path. A collection $\Gamma$ of rectifiable paths in $X$ is said to have zero $p$-modulus if there exists a Borel function $\rho \in L^{p}(X, \mu)$ with arbitrarily small $L^{p}$-norm such that

$$
\int_{\gamma} \rho d s \geq 1
$$

for all $\gamma \in \Gamma$. If inequality (2) is only assumed to hold except on a path family of zero $p$-modulus, then we say that $g$ is a $p$-weak upper gradient of $f$.

If $f$ is locally Lipschitz, then the upper local Lipschitz constant of $f$, defined by

$$
\operatorname{Lip}(f)(x)=\limsup \sup _{r \rightarrow 0} \frac{d_{Y}(f(x), f(y))}{r},
$$

is an upper gradient of $f$ [7, Proposition 1.11]. In fact, in this case, a slightly more delicate argument shows that the lower local Lipschitz constant of $f$, defined by

$$
\operatorname{lip}(f)(x)=\liminf _{r \rightarrow 0} \sup _{y \in B(x, r)} \frac{d_{Y}(f(x), f(y))}{r},
$$

is an upper gradient of $f$. Both statements may fail if $f$ is not assumed to be locally Lipschitz.

We next briefly discuss integration of metric space valued mappings. See [18, Sect. 2] and [41, Sect. 3.3] for a more detailed account, and in particular for a discussion of Bochner integrability. The following notion of local integrability of metric space valued mappings is perhaps not yet standard.

Definition 2.1 (locally integrable) A mapping $f: X \rightarrow Y$ is in the class $L_{\mathrm{loc}}^{1}(X ; Y)$, i.e., it is said to be locally integrable, if it is Bochner measurable and there exists a point $z \in Y$ such that the function $x \mapsto d_{Y}(f(x), z)$ is in the space $L_{\text {loc }}^{1}(X)$.

In the case that $Y$ is a Banach space, Definition 2.1 is equivalent to the condition that the mapping in question is locally Bochner integrable (see [18, Sect. 2]). For our purposes, it will be sufficient to note that if $X$ is a separable metric space, any continuous mapping from $\left(X, d_{X}, \mu\right)$ to another metric space is locally integrable.

Given a measurable subset $E$ of $X$ of finite and positive measure, a Banach space $V$, and a locally integrable mapping $f: X \rightarrow V$, we define the average value off on $E$ using the Bochner integral:

$$
f_{E}=f_{E} f d \mu=\frac{1}{\mu(E)} \int_{E} f d \mu .
$$

Fundamental work of Heinonen and Koskela has resulted in an analytic condition which guarantees the presence of "many" rectifiable curves in a metric space [16]. Fix 
$p \geq 1$. Let $V$ be a Banach space, let $f: X \rightarrow V$ be a locally integrable mapping, and let $g: X \rightarrow[0, \infty]$ be a measurable function. The pair $(f, g)$ satisfies a $p$-Poincaré inequality with constant $C>0$ and dilation factor $\sigma>0$ if for each ball $B$ in $X$,

$$
f_{B}\left|f-f_{B}\right| d \mu \leq C(\operatorname{diam} B)\left(f_{\sigma B} g^{p} d \mu\right)^{\frac{1}{p}} .
$$

The space $(X, d, \mu)$ supports a p-Poincaré inequality if there is a constant $C>0$ and a dilation factor $\sigma>0$ such that for any locally integrable function $f: X \rightarrow \mathbb{R}$ and each $p$-weak upper gradient $g$ of $f$, the pair $(f, g)$ satisfies a $p$-Poincaré inequality with constant $C$ and dilation factor $\sigma$.

By [18, Theorem 4.3] and [21, Theorem 2], if $X$ is complete, doubling and supports a $p$-Poincaré inequality, then for any metric space $Y$, any locally integrable mapping $f: X \rightarrow Y$, any $p$-weak upper gradient $g$ of $f$, and any isometric embedding $\iota$ of $Y$ into a Banach space $V$, the pair $(\iota \circ f, g)$ supports a $p$-Poincaré inequality, quantitatively.

\subsection{Measurable Differential Structures}

We briefly outline the theory of differentiation in metric spaces developed by Cheeger [7]. The interested reader could also see the work of Keith [22] and the primer of Kleiner and Mackay [24].

Definition 2.2 (measurable differentiable structure) A measurable differentiable structure on a complete metric measure space $(X, d, \mu)$ is a countable collection of pairs $\left\{\left(X_{\alpha}, \varphi_{\alpha}\right)\right\}_{\alpha \in I}$, called coordinate patches, that satisfy the following conditions:

- for each $\alpha \in I$, the set $X_{\alpha}$ is a measurable subset of $X$ of positive measure,

- the union $\bigcup_{\alpha} X_{\alpha}$ is pairwise disjoint and has full measure in $X$,

- each $\varphi_{\alpha}$ is an $N(\alpha)$-tuple of Lipschitz functions on $X$, for some $N(\alpha) \in \mathbb{N}$ that is bounded above independently of $\alpha$,

- for every Lipschitz function $f: X \rightarrow \mathbb{R}$, there exists a collection of measurable functions $\left\{\partial f / \partial \varphi_{n}^{\alpha}: X_{\alpha} \rightarrow \mathbb{R}\right\}_{\alpha \in I, n \in\{1, \ldots, N(\alpha)\}}$ such that

$$
\lim _{\substack{y \rightarrow x \\ y \in X \backslash\{x\}}} \frac{\left|f(y)-f(x)-\sum_{n=1}^{N(\alpha)}\left(\varphi_{n}^{\alpha}(y)-\varphi_{n}^{\alpha}(x)\right) \frac{\partial f}{\partial \varphi_{n}^{\alpha}}(x)\right|}{d(y, x)}=0
$$

for each $\alpha \in I$ and for $\mu$-almost-every point $x \in X_{\alpha}$, and moreover that this condition determines the collection $\left\{\partial f / \partial \varphi_{n}^{\alpha}\right\}$ uniquely up to sets of measure zero.

Definition 2.3 (differentiability) Let $\left\{\left(X_{\alpha}, \varphi_{\alpha}\right)\right\}_{\alpha \in I}$ be a measurable differentiable structure on a metric measure space $(X, d, \mu)$ and let $V$ be a Banach space. Given a measurable subset $S$ of $X$, a Bochner measurable mapping $f: X \rightarrow V$ is differentiable at $\mu$-almost-every point of $S$ if there exists a collection of measurable functions 
$\left\{\partial f / \partial \varphi_{n}^{\alpha}: X_{\alpha} \cap S \rightarrow V\right\}_{\alpha \in I, n \in\{1, \ldots, N(\alpha)\}}$ such that

$$
\lim _{\substack{y \rightarrow x \\ y \in X \backslash\{x\}}} \frac{\left\|f(y)-f(x)-\sum_{n=1}^{N(\alpha)}\left(\varphi_{n}^{\alpha}(y)-\varphi_{n}^{\alpha}(x)\right) \frac{\partial f}{\partial \varphi_{n}^{\alpha}}(x)\right\|_{V}}{d(y, x)}=0
$$

for each $\alpha \in I$ and for $\mu$ almost-every point $x \in S \cap X_{\alpha}$, and moreover that this condition determines the collection $\left\{\partial f / \partial \varphi_{n}^{\alpha}\right\}$ uniquely up to sets of measure zero in $S$.

We recall that a Banach space $V$ has the Radon-Nikodym property if every Lipschitz function $f: \mathbb{R} \rightarrow V$ is differentiable almost everywhere with respect to Lebesgue measure. The following theorem can be viewed as a Rademacher theorem for Banach space-valued mappings on metric spaces [9].

Theorem 2.4 (Cheeger-Kleiner) Let $(X, d, \mu)$ be a complete and doubling metric measure space that supports a p-Poincaré inequality for some $1 \leq p<\infty$, and let $\left\{\left(X_{\alpha}, \varphi_{\alpha}\right)\right\}_{\alpha \in I}$ be a measurable differentiable structure on $(X, d, \mu)$. Then every Lipschitz mapping from $X$ to a Banach space with the Radon-Nikodym property is differentiable almost everywhere in $X$.

\subsection{Lorentz Spaces}

We now introduce the Lorentz spaces, which refine the Lebesgue spaces.

Given a measure space $(X, \mu)$ and a Banach space $\left(V,\|\cdot\|_{V}\right)$, we denote by $\mathcal{M}_{0}$ the following class of functions:

$$
\mathcal{M}_{0}:=\left\{\|f\|_{V} \in \mathcal{M}: f \text { is } \mu \text {-measurable and finite } \mu \text {-almost everywhere }\right\} .
$$

Given $f \in \mathcal{M}_{0}$, we define the distribution function $\omega_{f}:[0, \infty) \rightarrow[0, \infty]$ and the nonincreasing rearrangement $f^{*}:[0, \infty) \rightarrow[0, \infty]$ by

$$
\begin{aligned}
\omega_{f}(\alpha) & :=\mu\left(\left\{x \in X:\|f(x)\|_{V}>\alpha\right\}\right), \\
f^{*}(t) & :=\inf \left\{\alpha \geq 0: \omega_{f}(\alpha) \leq t\right\} .
\end{aligned}
$$

Let $1 \leq Q \leq \infty$ and $0<q \leq \infty$. The $(Q, q)$ Lorentz class consists of those functions $f \in \mathcal{M}_{0}(X)$ such that the quantity

$$
\|f\|_{Q, q}:= \begin{cases}\left(\int_{0}^{\infty}\left(t^{1 / Q} f^{*}(t)\right)^{q} \frac{d t}{t}\right)^{1 / q}, & 0<q<\infty \\ \sup _{0<t<\infty}\left\{t^{1 / Q} f^{*}(t)\right\}, & Q<\infty \text { and } q=\infty, \\ f^{*}(0), & q=Q=\infty\end{cases}
$$

is finite. If $1 \leq q \leq Q$, then $\|\cdot\|_{Q, q}$ defines a semi-norm on the $(Q, q)$ Lorentz class, and the corresponding normed space $\left(L^{Q, q}(X),\|\cdot\|_{Q, q}\right)$ is a Banach space. We refer to it as the $(Q, q)$ Lorentz space. 


\section{The Proof of Theorem 1.2}

In this section, we assume the hypotheses of Theorem 1.2. Namely, we fix $Q \geq 1$ and $1 \leq q \leq Q$, and let $(X, d, \mu)$ be a complete and Ahlfors $Q$-regular metric space that supports a $q$-Poincaré inequality, and let $Y$ be any metric space. We consider a continuous and locally integrable function $f: X \rightarrow Y$ and the set

$$
E=\{x \in X: \operatorname{lip} f(x)=\infty\} .
$$

Let us consider the case $Q=1$, which implies that $q=1$. We assume that lip $f \in$ $L^{1,1}(X)=L^{1}(X)$, and that $E$ is countable. An easy extension of [42, Lemma 3.9] to include metric space valued mappings shows that lip $f$ is a 1 weak upper gradient of $f$ under precisely our assumptions. As shown in [25, Lemma 2.4], a 1-weak upper gradient can be approximated in $L^{1}(X)$ by a (non-weak) upper gradient. Hence there is an upper gradient of $f$ in every $L^{1}(X)$ neighborhood of lip $f$. Since $L^{1}(X)=L^{1,1}(X)$, this suffices.

We now assume that $Q>1$. In the case that $q>1$, the open-endedness result of Keith and Zhong [26, Theorem 1.0.1] states that $X$ actually supports a $q^{\prime}$ Poincaré inequality for some $q^{\prime}<q$, quantitatively. Since a set that has Hausdorff dimension at most $(Q-q)$ has zero $\left(Q-q^{\prime}\right)$-dimensional Hausdorff measure, we assume without loss of generality that $1 \leq q<Q$, that lip $f \in L^{Q, 1}(X)$, and that the set $E$ has $\sigma$-finite $(Q-q)$-dimensional Hausdorff measure. We wish to show that $f$ has an upper gradient in the space $L^{Q, 1}(X)$. Again, [42, Lemma 3.9] implies that lip $f$ is a $q$-weak upper gradient of $f$, and hence the pair $(f, \operatorname{lip} f)$ satisfies the $q$-Poincaré inequality.

It is a well-known principle that if a function-gradient pair $(f, g)$ satisfies the $q$ Poincaré inequality, then the perturbed maximal function

$$
M_{q}(g)(x):=\left(\sup _{r>0} f_{B(x, r)} g^{q} d \mu\right)^{1 / q}
$$

provides a pointwise bound almost everywhere on the oscillation of $f$, i.e., $M_{q}(g)$ is a Hajtasz upper gradient of $f$. See [17, Theorem 3.2] and [18, Proposition 4.6]. Accordingly, there is a constant $C \geq 1$, depending only on the data, and a set $N$ of $\mu$-measure zero such that for each pair of points $x, y \in X \backslash N$,

$$
d_{Y}(f(x), f(y)) \leq C d_{X}(x, y)\left(M_{q}(\operatorname{lip} f)(x)+M_{q}(\operatorname{lip} f)(y)\right) .
$$

The Hardy-Littlewood maximal function theorem [15, Theorem 2.2] and the Marcinkiewicz Interpolation Theorem [4, Theorem IV.4.13] can be shown to imply the boundedness of the operator $M_{q}: L^{Q, 1}(X) \rightarrow L^{Q, 1}(X)$. This was essentially stated in [35] and proven in detail in [40, Sect. 4].

Define $g: X \rightarrow[0, \infty]$ by

$$
g(x)= \begin{cases}C M_{q}(\operatorname{lip} f)(x) & x \notin N, \\ \infty & x \in N .\end{cases}
$$


Then

$$
\|g\|_{Q, 1} \lesssim\left\|M_{q}(\operatorname{lip} f)\right\|_{Q, 1} \lesssim\|\operatorname{lip} f\|_{Q, 1},
$$

and the inequality (6) implies that for every pair of points $x$ and $y$ in $X$,

$$
d_{Y}(f(x), f(y)) \leq d_{X}(x, y)(g(x)+g(y)) .
$$

A short argument, given in [37, Lemmas 4.6 and 4.7], now shows that $4 g$ is an upper gradient of $f$.

\section{The Proof of Theorem 1.5}

In order to prove Theorem 1.5, we first show that the mapping $f$ in question satisfies Lip $f(x)<\infty$ for almost every $x \in X$, and then apply a Stepanov-type theorem in the spirit of Cheeger and Kleiner. The first step follows from [41]. The second step is based on the Cheeger-Kleiner generalization of Rademacher's theorem (Theorem 2.4 above), and the usual method of proving Stepanov's theorem from Rademacher's theorem (see, for example, [12,3.1.9]). A key tool is an extension theorem for Lipschitz functions from a doubling metric space to a Banach space. Such results can already be deduced from the arguments in [20], and are certainly implied by the much stronger results of [27] and [28].

For a mapping $f: X \rightarrow Y$ between metric spaces, we denote

$$
S_{f}=\{x \in X: \operatorname{Lip} f(x)<\infty\} .
$$

See [12, 3.1.9] for a proof of the following standard lemma:

Lemma 4.1 Let $(X, d, \mu)$ be a separable metric measure space, $\left(Y, d_{Y}\right)$ be any metric space, and $f: X \rightarrow Y$ be a mapping. Then there is a sequence $\left\{C_{k}\right\}_{k \in \mathbb{N}}$ of closed subsets of $X$ with $S_{f}=\bigcup_{k \in \mathbb{N}} C_{k}$, such that for each $k \in \mathbb{N}$, there are numbers $r_{k}>0$ and $L_{k} \geq 1$ such that

- the restriction $\left.f\right|_{C_{k}}$ is $L_{k}$-Lipschitz, and

- if $x \in C_{k}$ and $y \in B\left(x, r_{k}\right)$, then

$$
d(f(x), f(y)) \leq L_{k} d(x, y) .
$$

Theorem 4.2 Let $(X, d, \mu)$ be a complete and doubling metric measure space satisfying a p-Poincaré inequality for some $1 \leq p<\infty$, and let $V$ be a Banach space with the Radon-Nikodym property. Then each measurable function $f: X \rightarrow V$ is differentiable at almost every point of the set $S_{f}=\{x \in X: \operatorname{Lip} f(x)<\infty\}$ with respect to any measurable differentiable structure on $X$.

Proof Let $\left\{C_{k}\right\}_{k \in \mathbb{N}}$ be the sequence of closed sets provided by Lemma 4.1, and let $\left\{r_{k}\right\}_{k \in \mathbb{N}}$ and $\left\{L_{k}\right\}_{k \in \mathbb{N}}$ be the associated sequences of radii and constants. Applying 
a suitable Lipschitz extension theorem (for example, [28, Theorem 1.5]), there is a Lipschitz mapping $F_{k}: X \rightarrow V$ such that the restriction of $F_{k}$ to $C_{k}$ agrees with $f$; after possibly increasing $L_{k}$, we may assume that $F_{k}$ is also $L_{k}$-Lipschitz.

Let $\left\{\left(X_{\alpha}, \varphi^{\alpha}\right)\right\}_{\alpha \in I}$ be a measurable differential structure on $X$. By the CheegerKleiner generalization of Rademacher's theorem [9, Theorem 1.5] (see Theorem 2.4), the mapping $F_{k}$ is differentiable at almost every point of $X$ with respect to this structure. Let

$$
\left\{\partial F_{k} / \partial \varphi_{n}^{\alpha}: X_{\alpha} \rightarrow V\right\}_{\alpha \in I, n=1, \ldots, N(\alpha)}
$$

be the collection of partial derivatives of $F_{k}$ over all coordinate patches.

In order to show the differentiability of $f$ almost everywhere in the set $S_{f}$, we will show that the countable collection

$$
\left\{\left.\left(\partial F_{k} / \partial \varphi_{n}^{\alpha}\right)\right|_{S_{f}}: S_{f} \cap X_{\alpha} \rightarrow V\right\}_{\alpha \in I, n=1, \ldots, N(\alpha)}
$$

consists of Borel measurable functions satisfying

$$
\limsup _{m \rightarrow \infty} \frac{\left\|f\left(y_{m}\right)-f\left(x_{0}\right)-\sum_{n=1}^{N(\alpha)}\left(\varphi_{n}^{\alpha}\left(y_{m}\right)-\varphi_{n}^{\alpha}\left(x_{0}\right)\right) \frac{\partial F_{k}}{\partial \varphi_{n}^{\alpha}}\left(x_{0}\right)\right\|_{V}}{d\left(y_{m}, x_{0}\right)}=0
$$

for each $\alpha \in I$, almost every point $x_{0}$ in $S_{f} \cap X_{\alpha}$, any sequence $\left\{y_{m}\right\}_{m \in \mathbb{N}}$ of points in $X \backslash\left\{x_{0}\right\}$ that converges to $x_{0}$, and moreover that up to a set of measure zero in $S_{f}$, the collection (9) is the only such collection satisfying (10).

The Borel measurability of the functions in (9) follows from the corresponding statement for the functions in (8) and the decomposition of $S_{f}$ into a countable union of closed sets. By this decomposition, the Lebesgue differentiation theorem [15, Theorem 1.8], and the almost everywhere differentiability of the functions $\left\{F_{k}\right\}$, we need only show (10) for a point $x_{0}$ that is both a density point of $C_{k} \cap X_{\alpha}$ and a point of differentiability of $F_{k}$, for some $k \in \mathbb{N}$ and $\alpha \in I$.

Let $\left\{y_{m}\right\}_{m \in \mathbb{N}} \subseteq X \backslash\left\{x_{0}\right\}$ be a sequence converging to $x_{0}$, and fix $0<\epsilon<1$. Using the argument from [3, Page 409] and Lemma 4.1, since $x_{0}$ is a density point for $C_{k} \cap X_{\alpha}$, we may find a radius $r_{0}>0$ such that if $y_{m} \in B\left(x_{0}, r_{0}\right)$, then there is a point $x_{m} \in C_{k} \cap X_{\alpha}$ satisfying

$$
\begin{aligned}
& d\left(y_{m}, x_{m}\right) \leq \epsilon d\left(y_{m}, x_{0}\right), \text { and } \\
& \left\|f\left(y_{m}\right)-f\left(x_{m}\right)\right\|_{V} \leq L_{k} d\left(x_{m}, y_{m}\right)
\end{aligned}
$$

For each $m \in \mathbb{N}$, define

$$
\begin{aligned}
& L_{1}(m)=\frac{\left\|f\left(y_{m}\right)-f\left(x_{m}\right)-\sum_{n=1}^{N(\alpha)}\left(\varphi_{n}^{\alpha}\left(y_{m}\right)-\varphi_{n}^{\alpha}\left(x_{m}\right)\right) \frac{\partial F_{k}}{\partial \varphi_{n}^{\alpha}}\left(x_{0}\right)\right\|_{V}}{d\left(y_{m}, x_{0}\right)}, \\
& L_{2}(m)=\frac{\left\|f\left(x_{m}\right)-f\left(x_{0}\right)-\sum_{n=1}^{N(\alpha)}\left(\varphi_{n}^{\alpha}\left(x_{m}\right)-\varphi_{n}^{\alpha}\left(x_{0}\right)\right) \frac{\partial F_{k}}{\partial \varphi_{n}^{\alpha}}\left(x_{0}\right)\right\|_{V}}{d\left(y_{m}, x_{0}\right)} .
\end{aligned}
$$


We claim that

$$
\limsup _{m \rightarrow \infty}\left(L_{1}(m)+L_{2}(m)\right)=0,
$$

which implies (10) by the triangle inequality. Again increasing $L_{k}$ if needed, we may assume that each chart mapping $\varphi_{n}^{\alpha}$ is also $L_{k}$-Lipschitz. By the triangle inequality, (11), (12), and the fact that $x_{m} \in C_{k}$,

$$
\begin{aligned}
L_{1}(m) & \leq \epsilon\left(\frac{\left\|f\left(y_{m}\right)-f\left(x_{m}\right)\right\|_{V}}{d\left(y_{m}, x_{m}\right)}+\frac{\left(\sum_{n=1}^{N(\alpha)}\left|\varphi_{n}^{\alpha}\left(y_{m}\right)-\varphi_{n}^{\alpha}\left(x_{m}\right)\right|\right)\left\|\frac{\partial F_{k}}{\partial \varphi_{n}^{\alpha}}\left(x_{0}\right)\right\|_{V}}{d\left(y_{m}, x_{m}\right)}\right) \\
& \leq \epsilon\left(L_{k}+\sum_{n=1}^{N(\alpha)} L_{k}\left\|\frac{\partial F_{k}}{\partial \varphi_{n}^{\alpha}}\left(x_{0}\right)\right\|_{V}\right)
\end{aligned}
$$

whenever $y_{m} \in B\left(x_{0}, r_{0}\right)$, i.e., for all sufficiently large $m$. Since the final quantity above tends to zero as $\epsilon$ tends to zero, this shows that $\lim _{\sup } \operatorname{si\infty }_{m} L_{1}(m)=0$.

A similar argument using the triangle inequality, (11), and the fact that $x_{m}$ and $x_{0}$ are points of $C_{k}$ imply that

$$
\begin{aligned}
L_{2}(m) \leq & \frac{\left\|f\left(x_{m}\right)-f\left(x_{0}\right)-\sum_{n=1}^{N(\alpha)}\left(\varphi_{n}^{\alpha}\left(x_{m}\right)-\varphi_{n}^{\alpha}\left(x_{0}\right)\right) \frac{\partial F_{k}}{\partial \varphi_{n}^{\alpha}}\left(x_{0}\right)\right\|_{V}}{d\left(x_{m}, x_{0}\right)} \\
& \cdot \frac{d\left(x_{m}, y_{m}\right)+d\left(y_{m}, x_{0}\right)}{d\left(y_{m}, x_{0}\right)} \\
\leq & \frac{\left\|F_{k}\left(x_{m}\right)-F_{k}\left(x_{0}\right)-\sum_{n=1}^{N(\alpha)}\left(\varphi_{n}^{\alpha}\left(x_{m}\right)-\varphi_{n}^{\alpha}\left(x_{0}\right)\right) \frac{\partial F_{k}}{\partial \varphi_{n}^{\alpha}}\left(x_{0}\right)\right\|_{V}}{d\left(x_{m}, x_{0}\right)} \cdot(1+\epsilon) .
\end{aligned}
$$

Since $F_{k}$ is differentiable at $x_{0}$, the above quantity tends to zero as $m$ tends to infinity. This completes the proof of (13) and hence of (10).

We now show that the collection (9) is unique up to a set of measure zero in $S_{f}$. By the uniqueness of the collection (8), it suffices to show that if $x_{0}$ is a point of density of some $C_{k} \cap X_{\alpha}$, and $\left\{g_{n}\right\}_{n=1}^{N(\alpha)}$ is a collection of Borel measurable functions satisfying the following implication: If $\left\{y_{m}\right\} \subseteq X$ is a sequence converging to $x_{0}$, then the validity of

$$
\limsup _{m \rightarrow \infty} \frac{\left\|f\left(y_{m}\right)-f\left(x_{0}\right)-\sum_{n=1}^{N(\alpha)}\left(\varphi_{n}^{\alpha}\left(y_{m}\right)-\varphi_{n}^{\alpha}\left(x_{0}\right)\right) g_{n}\left(x_{0}\right)\right\|_{V}}{d\left(y_{m}, x_{0}\right)}=0
$$

implies the validity of

$$
\limsup _{m \rightarrow \infty} \frac{\left\|F_{k}\left(y_{m}\right)-F_{k}\left(x_{0}\right)-\sum_{n=1}^{N(\alpha)}\left(\varphi_{n}^{\alpha}\left(y_{m}\right)-\varphi_{n}^{\alpha}\left(x_{0}\right)\right) g_{n}\left(x_{0}\right)\right\|_{V}}{d\left(y_{m}, x_{0}\right)}=0 .
$$

The proof of this is analogous to the proof of (10) and is left to the reader. 
Proof of Theorem 1.5 We fix $Q \geq 1$, and let $(X, d, \mu)$ be a complete and Ahlfors $Q$-regular metric space that supports a $Q$-Poincaré inequality. Moreover, let $V$ be a Banach space that has the Radon-Nikodym property, i.e., every Lipschitz function from $\mathbb{R}$ to $V$ is differentiable almost everywhere with respect to the Lebesgue measure on $\mathbb{R}$. We consider a continuous $f: X \rightarrow V$ that is assumed to have an upper gradient in the Lorentz space $L^{Q, 1}$.

It follows from [41, Corollary 6.7] (taking $f(X)$ as target space) that $f$ satisfies the $Q$-Rado-Reichelderfer condition, meaning that there is a number $\sigma>0$ and a non-negative function $\Theta \in L^{1}(X)$ such that for any ball $B$ in $X$,

$$
\operatorname{diam}(f(B))^{Q} \leq \int_{\sigma B} \Theta d \mu
$$

An easy computation now shows that Lip $f(x)<\infty$ for almost every $x \in X[41$, Proposition 6.4]. Theorem 4.2 completes the proof.

\section{Non-embedding Results}

We now apply the results of the previous sections to prove a non-embedding result, Theorem 1.6. The material in the preparatory first two subsections might be familiar to experts.

\subsection{Existence and Basic Properties of Weak Tangent Mappings}

We begin with the existence and properties of a weak tangent mapping of a bi-Lipschitz embedding. This construction is standard and can be accomplished in several different but equivalent ways; see, for example, [7] or [1]. We include it for convenience and because we will later refer to specific parts of the construction. We follow the method of David and Semmes [11]. This approach has the advantage of being concrete although somewhat cumbersome in notation; it uses the Assouad embedding theorem to transfer the abstract notion of pointed Gromov-Hausdorff convergence to the Euclidean setting. In Euclidean space, the relevant notions of convergence are as follows.

A sequence $\left\{F_{j}\right\}_{j \in \mathbb{N}}$ of non-empty closed subsets of $\mathbb{R}^{N}$ converges to a non-empty closed subset $F$ of $\mathbb{R}^{N}$ if for all $r>0$,

$$
\begin{aligned}
& \lim _{j \rightarrow \infty} \sup \left\{\operatorname{dist}(x, F): x \in F_{j} \cap B(0, r)\right\}=0, \text { and } \\
& \lim _{j \rightarrow \infty} \sup \left\{\operatorname{dist}\left(x, F_{j}\right): x \in F \cap B(0, r)\right\}=0,
\end{aligned}
$$

where we use the convention $\sup \emptyset=0$.

A sequence of functions $\left\{f_{j}: F_{j} \rightarrow \mathbb{R}^{N}\right\}_{j \in \mathbb{N}}$ converges to a function $f: F \rightarrow \mathbb{R}^{N}$ if the sequence of sets $\left\{F_{j}\right\}_{j \in \mathbb{N}}$ converges to the set $F$ and for every convergent 
sequence $\left\{x_{j} \in F_{j}\right\}_{j \in \mathbb{N}}$,

$$
f\left(\lim _{j \rightarrow \infty} x_{j}\right)=\lim _{j \rightarrow \infty} f_{j}\left(x_{j}\right) .
$$

We recall that a pointed metric space is a triple consisting of a set, a metric on the set, and a point in the set (called the base point). We abuse notation by denoting, for $N \in \mathbb{N}$, the pointed metric space $\left(\mathbb{R}^{N},\|\cdot\|_{\mathbb{R}^{N}}, 0\right)$ by $\mathbb{R}^{N}$. A pointed mapping package is a triple $(A, B, f)$ where $A$ and $B$ are pointed metric spaces, and $f$ is a function between the sets underlying $A$ and $B$ that respects the base point.

We now assume that $\left(X, d_{X}, x_{0}\right)$ is a complete and doubling pointed metric space, and that $C \subseteq X$ is a closed set containing $x_{0}$. We denote by $\left(V,\|\cdot\|_{V}\right)$ a Banach space. We consider an embedding $\iota: X \hookrightarrow V$, and we assume that there is a number $A \geq 1$ such that the restriction $\left.\iota\right|_{C}$ is an $A$-bi-Lipschitz embedding of $C$ into $V$.

Take any sequence of scales $\left\{r_{j}\right\}_{j \in \mathbb{N}}$ tending to zero. For each $j \in \mathbb{N}$, define pointed metric spaces

$$
\begin{aligned}
C_{j} & =\left(C, d_{X} / r_{j}, x_{0}\right), \\
\iota(C)_{j} & =\left(\iota(C),\|\cdot\|_{V} / r_{j}, \iota\left(x_{0}\right)\right) .
\end{aligned}
$$

Note that these spaces are again complete and doubling with a uniform constant. Gromov's compactness theorem, Lemma 8.13 in [11], implies that for some subsequence of the scales $\left\{r_{j}\right\}_{j \in \mathbb{N}}$ (which we do not relabel) the corresponding sequences of pointed metric spaces converge, and we denote the limit spaces by

$$
C_{x_{0}}=\left(C_{\infty}, d_{\infty}, x_{\infty}\right) \text { and } \iota(C)_{\iota\left(x_{0}\right)}=\left(\iota(C)_{\infty}, \rho_{\infty}, y_{\infty}\right),
$$

respectively. The space $C_{x_{0}}$ is called a weak tangent to $C$ at $x_{0}$, and $\iota(C)_{\iota\left(x_{0}\right)}$ is named analogously.

After passing to another subsequence of $\left\{r_{j}\right\}_{j \in \mathbb{N}}$ (again, without relabeling), we may assume that pointed mapping packages $\left\{\left(C_{j}, \iota(C)_{j}, \iota\right)\right\}_{j \in \mathbb{N}}$ converge to a pointed mapping package $\left(C_{x_{0}}, \iota(C)_{\iota\left(x_{0}\right)}, \iota_{\infty}\right)$; see Lemma 8.22 in [11].

The meaning of the above convergence is the following:

- there are dimensions $N, M \in \mathbb{N}$, exponents $\alpha, \beta \in(0,1]$, constants $K, L \geq 1$, sequences of pointed mapping packages

$$
\left\{\left(C_{j}, \mathbb{R}^{N}, f_{j}\right)\right\}_{j \in \mathbb{N}}, \quad\left\{\left(\iota(C)_{j}, \mathbb{R}^{M}, g_{j}\right)\right\}_{j \in \mathbb{N}},
$$

and "limit" pointed mapping packages

$$
\left(C_{x_{0}}, \mathbb{R}^{N}, f\right), \quad\left(\iota(C)_{\iota\left(x_{0}\right)}, \mathbb{R}^{M}, g\right),
$$

where each of $\left\{f_{j}\right\}_{j \in \mathbb{N}}$ and $f$ is $(\alpha, K)$-bi-Hölder, and each of $\left\{g_{j}\right\}_{j \in \mathbb{N}}$ and $g$ is $(\beta, L)$-bi-Hölder. 
- the sequences of subsets $\left\{f_{j}\left(C_{j}\right)\right\}_{j \in \mathbb{N}}$ and $\left\{g_{j}\left(\iota\left(C_{j}\right)\right)\right\}_{j \in \mathbb{N}}$ converge to $f\left(C_{x_{0}}\right)$ and $g\left(\iota(C)_{\iota\left(x_{0}\right)}\right)$, respectively,

- the maps

$$
\begin{gathered}
\frac{d_{X}\left(f_{j}^{-1}(\cdot), f_{j}^{-1}(\cdot)\right)}{r_{j}}: f_{j}\left(C_{j}\right) \times f_{j}\left(C_{j}\right) \rightarrow \mathbb{R}, \\
\frac{\left\|g_{j}^{-1}(\cdot)-g_{j}^{-1}(\cdot)\right\|_{V}}{r_{j}}: g_{j}\left(\iota(C)_{j}\right) \times g_{j}\left(\iota(C)_{j}\right) \rightarrow \mathbb{R}, \\
g_{j} \circ \iota \circ f_{j}^{-1}: f_{j}(C) \rightarrow g_{j}(\iota(C)),
\end{gathered}
$$

converge to

$$
\begin{gathered}
d_{\infty}\left(f^{-1}(\cdot), f^{-1}(\cdot)\right): f\left(C_{x_{0}}\right) \times f\left(C_{x_{0}}\right) \rightarrow \mathbb{R}, \\
\rho_{\infty}\left(g^{-1}(\cdot), g^{-1}(\cdot)\right): g\left(\iota(C)_{\iota\left(x_{0}\right)}\right) \times g\left(\iota(C)_{\iota\left(x_{0}\right)}\right) \rightarrow \mathbb{R}, \\
g \circ \iota_{\infty} \circ f^{-1}: f\left(C_{x_{0}}\right) \rightarrow g\left(\iota(C)_{\iota\left(x_{0}\right)}\right),
\end{gathered}
$$

respectively.

It can easily be seen that the limit map $\iota_{\infty}: C_{x_{0}} \rightarrow \iota(C)_{\iota\left(x_{0}\right)}$ is also an $A$-biLipschitz embedding. Moreover, the doubling condition on $X$, and hence $C$, persists in the limit: $C_{x_{0}}$ and thus $\iota(C)_{\iota\left(x_{0}\right)}$ are also doubling.

\subsection{Properties of Weak Tangent Mappings at Points of Differentiability}

We now additionally assume the existence of a measure $\mu$ on $\left(X, d_{X}\right)$ so that the resulting metric measure space $\left(X, d_{X}, \mu\right)$ has a measurable differentiable structure $\left\{\left(X_{\alpha}, \varphi_{\alpha}\right)\right\}_{\alpha \in I}$, and that the embedding $\iota: X \hookrightarrow V$ is differentiable almost everywhere in $C$, with differentials $\left\{\frac{\partial \iota}{\partial \varphi_{n}^{\alpha}}:\left(C \cap X_{\alpha}\right) \rightarrow V\right\}_{\alpha \in I, n \in\{1, \ldots, N(\alpha)\}}$. Recall that this means that for each $\alpha \in I$ and $\mu$-almost every point $x_{0} \in C \cap X_{\alpha}$,

$$
\lim _{\substack{x \rightarrow x_{0} \\ x \in X \backslash\left\{x_{0}\right\}}} \frac{\left|\iota(x)-\iota\left(x_{0}\right)-\sum_{n=1}^{N(\alpha)}\left(\varphi_{n}^{\alpha}(x)-\varphi_{n}^{\alpha}\left(x_{0}\right)\right) \frac{\partial \iota}{\partial \varphi_{n}^{\alpha}}\left(x_{0}\right)\right|}{d\left(x, x_{0}\right)}=0,
$$

and moreover that this condition determines the collection $\left\{\frac{\partial \iota}{\partial \varphi_{n}^{\alpha}}\right\}_{n=1, \ldots, N(\alpha)}$ uniquely up to sets of measure zero in $C \cap X_{\alpha}$.

The following proposition, which follows immediately from the definitions, says that given a point $x_{0} \in C \cap X_{\alpha}$ at which (16) holds, the image $\iota(C)$ is contained up to first order in the span of the differentials of $\iota$ at $x_{0}$, a concept analogous to definitions introduced by Cheeger and Kleiner [8]. We denote by $F_{x_{0}}$ the span in $V$ of the vectors $\left\{\frac{\partial \iota}{\partial \varphi_{n}^{\alpha}}\left(x_{0}\right)\right\}_{n=1, \ldots, N(\alpha)}$. 
Proposition 5.1 Let $x_{0} \in C \cap X_{\alpha}$ be a point at which (16) holds. Then

$$
\limsup _{r \rightarrow 0} \sup _{x \in B_{X}\left(x_{0}, r\right) \cap C} \frac{\operatorname{dist}_{V}\left(\iota(x)-\iota\left(x_{0}\right), F_{x_{0}}\right)}{r}=0 .
$$

We now use this information to bound the dimension of the tangent spaces to $C$ by the dimension of the differentiable structure on $X$. When $C=X$ this type of result can be found in [7, Sect. 14] and is also mentioned in [8], although we have not been able to find an explicit proof of the proposition below in the literature.

Proposition 5.2 Let $x_{0} \in C \cap X_{\alpha}$ be a point at which (16) holds. Then the weak tangent space $\iota(C)_{\iota\left(x_{0}\right)}$ isometrically embeds into $F_{x_{0}}$.

Proof Let $y$ be a point in the weak tangent space $\iota(C)_{\iota\left(x_{0}\right)}$. By definition, we may find a sequence of points $x_{j} \in C$ such that $\lim _{j \rightarrow \infty} g_{j}\left(\iota\left(x_{j}\right)\right)=g(y) \in \mathbb{R}^{M}$. As the mapping in a pointed mapping package respects base points, $g_{j}\left(\iota\left(x_{0}\right)\right)=0 \in \mathbb{R}^{M}$ for all $j \in \mathbb{N}$. This implies that

$$
\|g(y)\|_{\mathbb{R}^{M}}=\lim _{j \rightarrow \infty}\left\|g_{j}\left(\iota\left(x_{j}\right)\right)-g_{j}\left(\iota\left(x_{0}\right)\right)\right\|_{\mathbb{R}^{M}} \geq \lim _{j \rightarrow \infty} \frac{1}{L}\left(\frac{\left\|\iota\left(x_{j}\right)-\iota\left(x_{0}\right)\right\|_{V}}{r_{j}}\right)^{\beta} .
$$

This shows that the sequence $\left\{\frac{\iota\left(x_{j}\right)-\iota\left(x_{0}\right)}{r_{j}}\right\}_{j \in \mathbb{N}}$ is bounded in $\left(V,\|\cdot\|_{V}\right)$. Moreover, there is a quantity $c=c(A, L, \beta, y)$, such that for all $j \in \mathbb{N}$,

$$
d_{X}\left(x_{j}, x_{0}\right) \leq c r_{j}
$$

Let $k \in \mathbb{N}$. Proposition 5.1 now implies the existence of a positive integer $J(k)$ such that for all $j \geq J(k)$, there is a point $y_{j} \in F_{x_{0}}$ satisfying

$$
\left\|\iota\left(x_{j}\right)-\iota\left(x_{0}\right)-y_{j}\right\|_{V} \leq \frac{1}{k} r_{j}
$$

Without loss of generality, we may assume that $J(k)<J(k+1)$ for all $k \in \mathbb{N}$. By passing to a subsequence, we may further assume that $r_{j}=r_{J(j)}$. The triangle inequality now implies that the sequence $\left\{y_{j} / r_{j}\right\}_{j \in \mathbb{N}}$ is bounded. As each element of the sequence is in the finite-dimensional subspace $F_{x_{0}}$, after passing to a subsequence we may assume that $\left\{y_{j} / r_{j}\right\}_{j \in \mathbb{N}}$ converges to a point $\bar{y} \in F_{x_{0}}$. It now follows from (17) that

$$
\lim _{j \rightarrow \infty} \frac{\iota\left(x_{j}\right)-\iota\left(x_{0}\right)}{r_{j}}=\bar{y} \in F_{x_{0}}
$$

as well.

We wish to define a map $G: \iota(C)_{\iota\left(x_{0}\right)} \rightarrow F_{x_{0}}$ by $G(y)=\bar{y}$. Unfortunately, given points $y$ and $w$ in $\iota(C)_{\iota(x)}$, the subsequences of the scales $\left\{r_{j}\right\}_{j \in \mathbb{N}}$ used to define $\bar{y}$ and $\bar{w}$ might not coincide. We overcome this using the separability of the doubling space 
$\iota(C)_{\iota\left(x_{0}\right)}$. Let $\left\{z_{i}\right\}_{i \in \mathbb{N}}$ be a countable dense set in $\iota(C)_{\iota\left(x_{0}\right)}$. As argued above, we may find an infinite subset $S_{1} \subseteq \mathbb{N}$, a sequence $\left\{x_{j}^{1}\right\}_{j \in S_{1}} \subseteq C$, and a point $\bar{z}_{1} \in F_{x_{0}}$ such that

$$
g\left(z_{1}\right)=\lim _{\substack{j \rightarrow \infty \\ j \in S_{1}}} g_{j}\left(\iota\left(x_{j}^{1}\right)\right) \text { and } \lim _{\substack{j \rightarrow \infty \\ j \in S_{1}}} \frac{\iota\left(x_{j}^{1}\right)-\iota\left(x_{1}\right)}{r_{j}}=\bar{z}_{1} \in F_{x_{0}} .
$$

We now define $G\left(z_{1}\right)=\bar{z}_{1}$. We continue inductively, defining $G\left(z_{i+1}\right)$ similarly but requiring that $S_{i+1} \subseteq S_{i}$.

We now check that $G$ is an isometric embedding of the set $\left\{z_{i}\right\}_{i \in \mathbb{N}}$ into $F_{x_{0}}$. Choose $i<k$ in $\mathbb{N}$, and let $\left\{x_{j}^{i}\right\}_{j \in S_{i}}$ and $\left\{x_{j}^{k}\right\}_{j \in S_{k}}$ be the sequences in $C$ used to define $G\left(z_{i}\right)$ and $G\left(z_{k}\right)$ respectively. Since $S_{k} \subseteq S_{i}$, we have

$$
\begin{aligned}
& g\left(z_{i}\right)=\lim _{\substack{j \rightarrow \infty \\
j \in S_{k}}} g_{j}\left(\iota\left(x_{j}^{i}\right)\right) \text { and } G\left(z_{i}\right)=\lim _{\substack{j \rightarrow \infty \\
j \in S_{k}}} \frac{\iota\left(x_{j}^{i}\right)-\iota\left(x_{0}\right)}{r_{j}}, \\
& g\left(z_{k}\right)=\lim _{\substack{j \rightarrow \infty \\
j \in S_{k}}} g_{j}\left(\iota\left(x_{j}^{k}\right)\right) \text { and } G\left(z_{k}\right)=\lim _{\substack{j \rightarrow \infty \\
j \in S_{k}}} \frac{\iota\left(x_{j}^{k}\right)-\iota\left(x_{0}\right)}{r_{j}} .
\end{aligned}
$$

Since the functions

$$
\left\{\frac{\left\|g_{j}^{-1}(\cdot)-g_{j}^{-1}(\cdot)\right\|_{V}}{r_{j}}: g_{j}\left(\iota(C)_{j}\right) \times g_{j}\left(\iota(C)_{j}\right) \rightarrow \mathbb{R}\right\}_{j \in \mathbb{N}}
$$

converge to the function

$$
\rho_{\infty}\left(g^{-1}(\cdot), g^{-1}(\cdot)\right): g\left(\iota(C)_{\iota\left(x_{0}\right)}\right) \times g\left(\iota(C)_{\iota\left(x_{0}\right)}\right) \rightarrow \mathbb{R}
$$

we see that

$$
\rho_{\infty}\left(z_{i}, z_{k}\right)=\lim _{\substack{j \rightarrow \infty \\ j \in S_{k}}} \frac{\left\|\iota\left(x_{j}^{i}\right)-\iota\left(x_{j}^{k}\right)\right\|_{V}}{r_{j}}=\left\|G\left(z_{i}\right)-G\left(z_{k}\right)\right\|_{V},
$$

showing that $G$ is an isometric embedding. Since $\left\{z_{i}\right\}_{i \in \mathbb{N}}$ is dense in $\iota(C)_{\iota\left(x_{0}\right)}$, the map $G$ extends uniquely to an isometric embedding of $\iota(C)_{\iota\left(x_{0}\right)}$ into the complete space $F_{x_{0}}$.

Since the tangent mapping $\iota_{\infty}: C_{x_{0}} \rightarrow \iota(C)_{\iota\left(x_{0}\right)}$ induced by $\iota: C \hookrightarrow V$ is biLipschitz, we may record the following corollary.

Corollary 5.3 Let $x_{0} \in C \cap X_{\alpha}$ be a point at which (16) holds. Then the weak tangent space $C_{x_{0}}$ admits a bi-Lipschitz embedding into $F_{x_{0}}$.

We note that in fact the crucial property of $\iota$ used in proving Proposition 5.2 and Corollary 5.3 was not the differentiability (16) at the point $x_{0}$, but rather the fact that the image $\iota(C)$ is contained up to first order at $x_{0}$ in a finite-dimensional space. 


\subsection{The Proof of Theorem 1.6}

We consider a complete and Ahlfors $Q$-regular metric space $(X, d, \mu)$ that supports a $Q$-Poincaré inequality, $Q \geq 1$, and a Banach space $V$ with the Radon-Nikodym property. We equip $(X, d, \mu)$ with a measurable differentiable structure $\left.\left\{\left(X_{\alpha}, \varphi_{\alpha}\right)\right\}\right)_{\alpha \in I}$.

Let $\iota: X \rightarrow V$ be an embedding with an upper gradient in the Lorentz space $L^{Q, 1}(X)$. As discussed in the proof of Theorem 1.5, results in [41, Sect. 6] imply that

$$
\mathcal{H}_{X}^{Q}\left(X \backslash \mathcal{S}_{\iota}\right)=0 \text { and } \mathcal{H}_{V}^{Q}\left(\iota(X) \backslash \iota\left(\mathcal{S}_{\iota}\right)\right)=0
$$

In addition, the hypotheses of Theorem 1.6 state that

$$
\mathcal{H}_{V}^{Q}\left(\iota(X) \backslash \mathcal{S}_{\iota^{-1}}\right)=0 \text { and } \mathcal{H}_{X}^{Q}\left(X \backslash \iota^{-1}\left(\mathcal{S}_{\iota^{-1}}\right)\right)=0 .
$$

By Lemma 4.1, we may find a countable collection of closed sets $\left\{C_{k}\right\}_{k \in \mathbb{N}}$ in $X$ such that $\left.\iota\right|_{C_{k}}$ is Lipschitz and

$$
\mathcal{S}_{\iota}=\bigcup_{k \in \mathbb{N}} C_{k} .
$$

Since $X$ is doubling and hence separable, the space $\iota(X)$ is also separable. Again using Lemma 4.1, we find a countable collection of closed sets $\left\{D_{l}\right\}_{l \in \mathbb{N}}$ in $Y$ such that $\left.\iota^{-1}\right|_{D_{l}}$ is Lipschitz and

$$
\mathcal{S}_{l^{-1}}=\bigcup_{l \in \mathbb{N}} D_{l}
$$

Fix $k \in \mathbb{N}$. We note that $\iota\left(C_{k}\right)$ is closed in the separable space $\iota(X)$, as $\iota$ is an embedding. For each $l \in \mathbb{N}$, set

$$
D_{k, l}=\iota\left(C_{k}\right) \cap D_{l} \text { and } C_{k, l}=\iota^{-1}\left(D_{k, l}\right) .
$$

Then, for each pair $k, l \in \mathbb{N}$, the set $C_{k, l}$ is closed and $\left.\iota\right|_{C_{k, l}}$ is bi-Lipschitz. Moreover, (19) and (20) imply that

$$
\mathcal{H}_{X}^{Q}\left(X \backslash \bigcup_{k, l \in \mathbb{N}} C_{k, l}\right)=0
$$

Let $\left(X_{\alpha}, \varphi_{\alpha}\right)$ be a coordinate patch of minimal dimension $N(\alpha)=N$. Then we may find $k, l \in \mathbb{N}$ so that

$$
\mathcal{H}_{X}^{Q}\left(C_{k, l} \cap X_{\alpha}\right)>0
$$

Theorem 4.2 implies that $\iota$ is differentiable at almost every point $\mathcal{S}_{\iota}$, and so we may find a point $x_{0} \in C_{k, l} \cap X_{\alpha}$ that is both a density point of $C_{k, l}$ and a point of differentiability of $\iota$. 
From the discussion in Sect. 5.1, we may find some sequence of scales $\left\{r_{j}\right\}$ tending to zero such that the pointed metric spaces

$$
X_{j}=\left(X, d_{X} / r_{j}, x_{0}\right)
$$

converge in the sense described in Sect. 5.1 to a weak tangent which we denote by $X_{x_{0}}$. Since $X$ is Ahlfors $Q$-regular, it follows that $X_{x_{0}}$, when equipped with the $Q$ dimensional Hausdorff measure, is Ahlfors $Q$-regular as well [11, Lemma 9.7].

For ease of notation, we denote $C_{k, l}$ simply by $C$. Define pointed metric spaces

$$
\begin{aligned}
C_{j} & =\left(C, d_{X} / r_{j}, x_{0}\right), \\
\iota(C)_{j} & =\left(\iota(C),\|\cdot\|_{V} / r_{j}, \iota\left(x_{0}\right)\right) .
\end{aligned}
$$

As discussed in Sect. 5.1, after passing to a subsequence of the scales $\left\{r_{j}\right\}$ (which we do not re-label), the pointed mapping packages $\left\{\left(C_{j}, \iota(C)_{j}, \iota\right)\right\}_{j \in \mathbb{N}}$ converge to a pointed mapping package $\left(C_{x_{0}}, \iota(C)_{\iota\left(x_{0}\right)}, \iota_{\infty}\right)$. Moreover, the mapping $\iota_{\infty}$ is biLipschitz. By [11, Lemmas 9.12 and 9.13], the fact that $x_{0}$ is a density point of $C$ implies that $C_{x_{0}}$ and $X_{x_{0}}$ are isometric. Hence, $C_{x_{0}}$ is also Ahlfors $Q$-regular when equipped with the $Q$-dimensional Hausdorff measure. Corollary 5.3 now implies that $C_{x_{0}}$ isometrically embeds into the span of the differentials of $\iota$ at $x_{0}$, which is a vector space of dimension at most $N$. This implies that $Q \leq N$, as desired.

\section{The Sharpness of Theorem 1.2}

In this section we prove Theorem 1.4. For ease of notation we denote the dimension of the ambient cube by $N \geq 2$, and instead use $n \in \mathbb{N}$ as an iterative index.

Our construction requires a preliminary result regarding the capacity of a point.

Proposition 6.1 Let $\mathcal{S}$ be a rearrangement invariant Banach function space on $\mathbb{R}^{N}$ containing a compactly supported function $g \notin L^{N, 1}\left(\mathbb{R}^{N}\right)$. Then for all points a $\in \mathbb{R}^{N}$, $\epsilon>0$ and all $\tau \in[0,1]$, there is a Lipschitz function $\phi: \mathbb{R}^{N} \rightarrow[0, \tau]$ satisfying

- the support of $\phi$ is a compact subset of $B(a, \epsilon)$,

- $\phi$ is constant with value $\tau$ on a neighborhood of the point a,

- $\|\operatorname{Lip} \phi\|_{\mathcal{S}} \leq \epsilon$.

Proof It suffices to consider the case that $\tau=1$ and $a=0$. Let $g: \mathbb{R}^{N} \rightarrow \mathbb{R}$ be a compactly supported function in $\mathcal{S} \backslash L^{N, 1}\left(\mathbb{R}^{N}\right)$; denote the support of $g$ by $K$. Then

$$
\|g\|_{L^{N, 1}}=\int_{0}^{\mathcal{L}^{N}(K)} t^{\frac{1}{N}-1} g^{*}(t) d t=\infty .
$$

Define $u:\left[0, \mathcal{L}^{N}(K)\right] \rightarrow \mathbb{R}$ by

$$
u(r)=\int_{r}^{\mathcal{L}^{N}(K)} t^{\frac{1}{N}-1} g^{*}(t) d t .
$$


Since $g \in \mathcal{S}$, the axioms of a Banach function space imply that it is finite $\mu$-almost everywhere. Hence $g^{*}(r)<\infty$ for each $r>0$, and so the non-increasing property of $g^{*}$ implies that

$$
u(0)-u(r)=\infty
$$

for every $r>0$.

Fix $\epsilon>0$, and denote the volume of the unit ball in $\mathbb{R}^{N}$ by $\Omega_{N}$. For $0<\delta<\epsilon / 2$, define $\phi_{\epsilon, \delta}: B(0, \epsilon) \rightarrow[0,1]$ by

$$
\phi_{\epsilon, \delta}(x)= \begin{cases}1 & 0 \leq|x| \leq \delta \\ \lambda u\left(\Omega_{N}|x|^{N}\right)-\Lambda & \delta \leq|x| \leq \epsilon / 2 \\ 0 & |x|>\epsilon / 2\end{cases}
$$

where $\lambda, \Lambda \geq 0$ are chosen to make $\phi_{\epsilon, \delta}$ continuous, i.e.,

$$
\lambda=\left(u\left(\Omega_{N} \delta^{N}\right)-u\left(\Omega_{N}(\epsilon / 2)^{N}\right)\right)^{-1} \text {, and } \Lambda=\lambda u\left(\Omega_{N}(\epsilon / 2)^{N}\right) .
$$

A calculation shows that $\phi_{\epsilon, \delta}$ is Lipschitz, and that there is a constant $C>0$ depending only on $N$ such that for almost every $x \in \mathbb{R}^{N}$,

$$
\operatorname{Lip} \phi_{\epsilon, \delta}(x) \leq \begin{cases}C \lambda g^{*}\left(\Omega_{N}|x|^{N}\right) & \delta \leq|x| \leq \epsilon / 2 \\ 0 & \text { otherwise. }\end{cases}
$$

Another calculation shows that the functions $x \in \mathbb{R}^{N} \mapsto g^{*}\left(\Omega_{N}|x|^{N}\right)$ and $t \in[0, \infty) \mapsto g^{*}(t)$ have the same distribution functions. Since $\mathcal{S}$ is rearrangement invariant, it follows that the function $x \in \mathbb{R}^{N} \mapsto g^{*}\left(\Omega_{N}|x|^{N}\right)$ has finite $\mathcal{S}$-norm, and so

$$
\left\|\operatorname{Lip} \phi_{\epsilon, \delta}\right\|_{\mathcal{S}} \leq C \lambda\left\|g^{*}\left(\Omega_{N}|\cdot|^{N}\right)\right\|_{\mathcal{S}}
$$

By (21) and (22), the quantity $\lambda$ tends to 0 as $\delta$ tends to 0 . Thus, for sufficiently small $\delta$, the function $\phi_{\epsilon, \delta}$ satisfies the stated requirements.

We also require one elementary lemma for the proof of Theorem 1.4.

Lemma 6.2 There exists a sequence $\left\{a_{n}\right\}_{n \in \mathbb{N}}$ of positive real numbers such that

$$
\prod_{n=0}^{\infty}\left(\left(1-a_{n}\right)^{N}-\left(2 a_{n}\right)^{N}\right)>0 \text {. }
$$

Proof Noting that

$$
\lim _{x \rightarrow 0^{+}}(1-x)^{N}-(2 x)^{N}=1
$$


we may choose the sequence $\left\{a_{n}\right\}$ so that for each $n \in \mathbb{N}$

$$
\left(1-a_{n}\right)^{N}-\left(2 a_{n}\right)^{N}>e^{-2^{-n}}
$$

The desired inequality now follows from the calculation that

$$
\begin{aligned}
\log \left(\prod_{n=0}^{\infty}\left(\left(1-a_{n}\right)^{N}-\left(2 a_{n}\right)^{N}\right)\right) & =\sum_{n=0}^{\infty} \log \left(\left(1-a_{n}\right)^{N}-\left(2 a_{n}\right)^{N}\right) \\
& >-\sum_{n=0}^{\infty} 2^{-n}>-\infty
\end{aligned}
$$

Proof of Theorem 1.4 Let $\left\{j_{n}\right\}_{n \in \mathbb{N}}$ be a sequence of positive integers satisfying

$$
j_{0}=0, j_{n} \equiv 0 \bmod 3, \text { and } j_{n+1} \geq 9\left(j_{n}+1\right) .
$$

Set

$$
k_{n}=\frac{2 j_{n}}{3} \text { and } l_{n}=\frac{j_{n+1}}{3}+\frac{2 j_{n}}{3}+1 .
$$

By choosing the sequence $\left\{j_{n}\right\}$ to grow fast enough, we may assume that for each $n \in \mathbb{N}$,

$$
2^{j_{n}-l_{n}}=2^{-\frac{1}{3}\left(j_{n+1}-j_{n}\right)-1} \leq a_{n},
$$

where $\left\{a_{n}\right\}_{n \in \mathbb{N}}$ is the sequence from Lemma 6.2.

We use these parameters to define a sequence of collections of dyadic cubes in $[-1,1]^{N}$. Let

$$
Q_{0}=[-1,1]^{N} \text { and } \mathcal{Q}_{0}=\left\{Q_{0}\right\} \text {. }
$$

Fix $n \in \mathbb{N}$, and assume that the collection of $\mathcal{Q}_{n}$ of cubes of side-length $2 \cdot 2^{-j_{n}}$ has been defined. Given $Q \in \mathcal{Q}_{n}$ with center denoted by $a$, consider the standard subdivision of $Q$ into essentially disjoint cubes of side-length $2 \cdot 2^{-j_{n+1}}$. We declare such a sub-cube to be an element of the collection $\mathcal{Q}(Q)$ if its center $a^{\prime}$ satisfies

$$
2\left(2^{-l_{n}}\right)+2^{-j_{n+1}} \leq\left\|a-a^{\prime}\right\|_{\infty} \leq 2^{-j_{n}}-2^{-l_{n}}-2^{-j_{n+1}}
$$

The set $\mathcal{Q}(Q)$ is non-empty if the difference between the upper and lower bounds above is at least $2^{1-j_{n+1}}$. A calculation using the estimate $j_{n+1} \geq 9\left(j_{n}+1\right)$ shows that this is always the case. We inductively define

$$
\mathcal{Q}_{n+1}=\left\{\mathcal{Q}(Q): Q \in \mathcal{Q}_{n}\right\}
$$

Given a cube $Q \in \mathcal{Q}_{n}$, we denote the concentric sub-cube of side-length $2 \cdot 2^{-l_{n}}$ by $I_{Q}$. Note that for any $Q \in \mathcal{Q}_{n}$, the cube $I_{Q}$ is disjoint from each cube in $\mathcal{Q}_{n+1}$. Hence, 


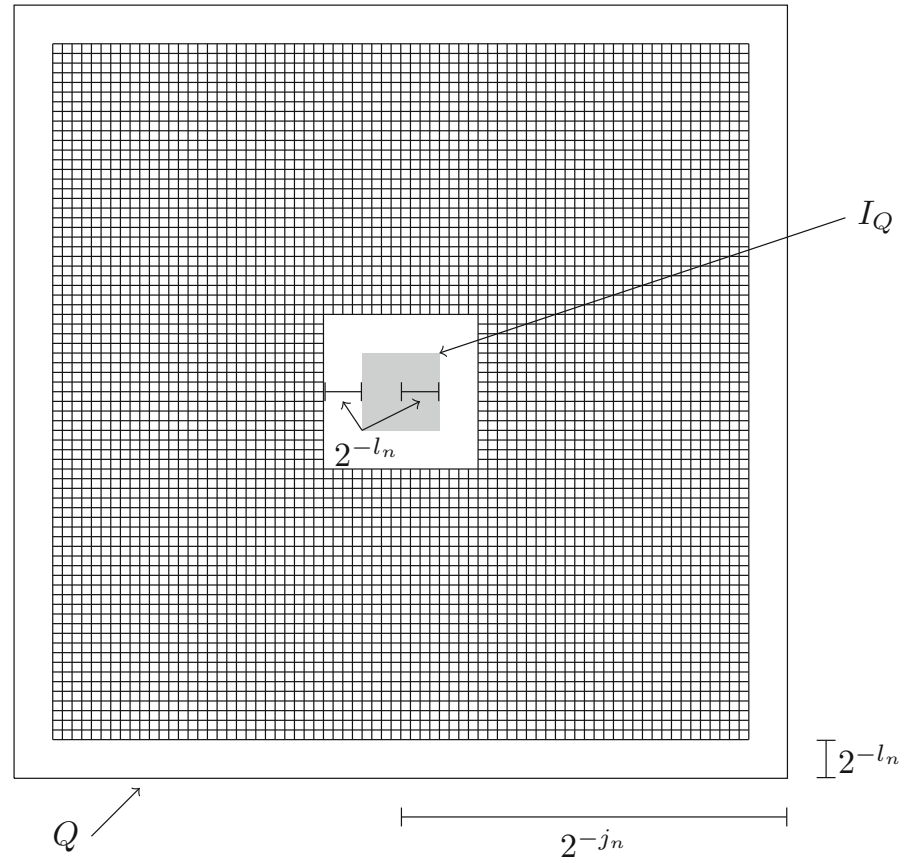

Fig. 1 A cube $Q \in \mathcal{Q}_{n}$, shown with the collection of subcubes $\mathcal{Q}(Q)$

given positive integers $n$ and $m$ and cubes $Q \in \mathcal{Q}_{n}$ and $Q^{\prime} \in \mathcal{Q}_{m}$, the set $I_{Q} \cap I_{Q^{\prime}}$ is empty unless $Q=Q^{\prime}$. See Fig. 1 .

For each $n \in \mathbb{N}$, fix a number $\epsilon_{n}>0$ so small that

$$
\left(\operatorname{card} \mathcal{Q}_{n}\right) \cdot \epsilon_{n} \leq 2^{-n}
$$

By Proposition 6.1, for each $Q \in \mathcal{Q}_{n}$, there is a Lipschitz function

$$
\phi_{Q}: \mathbb{R}^{N} \rightarrow\left[0,2^{-k_{n}}\right]
$$

with the following properties:

- $\phi_{Q} \equiv 2^{-k_{n}}$ on a neighborhood of the center of $I_{Q}$,

- $\phi_{Q} \equiv 0$ on a neighborhood of the boundary of $I_{Q}$,

- $\left\|\operatorname{Lip} \phi_{Q}\right\|_{\mathcal{S}} \leq \epsilon_{n}$.

We define a function $f: \mathbb{R}^{N} \rightarrow \mathbb{R}$ by declaring that for each $n \in \mathbb{N}$ and $Q \in \mathcal{Q}_{n}$,

$$
\left.f\right|_{I_{Q}}=\phi_{Q}
$$

and setting $f \equiv 0$ on the remaining subset of $\mathbb{R}^{N}$. 
We first claim that Lip $f(x)<\infty$ for every $x \in \mathbb{R}^{N}$. Set

$$
\begin{aligned}
& \mathcal{F}=\left\{x \in \mathbb{R}^{N}: \text { there is a largest } n \in \mathbb{N} \text { such that } x \in \bigcup_{Q \in \mathcal{Q}_{n}} Q\right\}, \\
& \mathcal{I}=\left\{x \in \mathbb{R}^{N}: x \in \bigcup_{Q \in \mathcal{Q}_{n}} Q^{0} \text { for every } n \in \mathbb{N}\right\},
\end{aligned}
$$

where $Q^{0}$ denotes the interior of the cube $Q$. Note that these sets partition $\mathbb{R}^{N}$ by construction.

If $x \in \mathcal{F}$ then either $f$ is identically zero in a neighborhood of $x$ or $f \equiv \phi_{Q^{\prime}}$ on a neighborhood of $x$, for some $Q^{\prime} \in \mathcal{Q}_{m}, m \in \mathbb{N}$. In either case, lip $f(x)<\infty$.

If $x \in \mathcal{I}$, and $Q \in \mathcal{Q}_{n}$ contains $x$, then

$$
\operatorname{dist}\left(x, \bigcup_{i=0}^{n} \bigcup_{Q \in \mathcal{Q}_{i}} I_{Q}\right) \geq 2^{-l_{n}} .
$$

Thus, if $y \in \mathbb{R}^{N}$ satisfies $|x-y|<2^{-l_{n}}$, then $|f(x)-f(y)| \leq 2^{-k_{n+1}}$. This implies that

$$
\operatorname{lip} f(x) \leq \liminf _{n \rightarrow \infty} \frac{2^{-k_{n+1}}}{2^{-l_{n}}}=0 .
$$

From the above discussion, we see that for all $x \in \mathbb{R}^{N}$,

$$
\operatorname{lip} f(x)=\sum_{n=0}^{\infty} \sum_{Q \in \mathcal{Q}_{n}} \operatorname{lip} \phi_{Q}(x),
$$

and thus, by the choice of the sequence $\left\{\epsilon_{n}\right\}_{n \in \mathbb{N}}$,

$$
\|\operatorname{lip} f\|_{\mathcal{S}} \leq 2 \text {. }
$$

We now show that $f$ fails to be differentiable at any point $x \in \mathcal{I}$. For each $n \in \mathbb{N}$, we may find a cube $Q \in \mathcal{Q}_{n}$ containing $x$ in its interior; denote the center of $Q$ by $a$. Let $b$ be the point of intersection of the boundary of $Q$ and the ray emanating from $a$ passing through $x$. Then

$$
\frac{|f(a)-f(b)|}{\|a-b\|} \geq \frac{2^{-k_{n}}}{\sqrt{N} \cdot 2^{-j_{n}}}=\frac{2^{j_{n} / 3}}{\sqrt{N}} .
$$

The triangle inequality and the collinearity of $a, b$, and $x$ now imply that there is a point $y \in\{a, b\}$ satisfying

$$
\frac{|f(x)-f(y)|}{\|x-y\|} \geq \frac{2^{j_{n} / 3}}{\sqrt{N}} .
$$


It follows that

$$
\operatorname{lip} f(x) \geq \limsup _{n \rightarrow \infty} \frac{2^{j_{n} / 3}}{2 \sqrt{N}}=\infty
$$

This implies that $f$ is not differentiable at $x$.

Finally, we show that $\mathcal{I}$ has positive $N$-dimensional Hausdorff measure. First, note that for any $n \in \mathbb{N}$ and $Q \in \mathcal{Q}_{n}$,

$$
\begin{aligned}
\mathcal{H}^{N}(Q) & =\left(2 \cdot 2^{-j_{n}}\right)^{N} \text { and } \mathcal{H}^{N}\left(\bigcup_{Q^{\prime} \in \mathcal{Q}(Q)} Q^{\prime}\right) \\
& =\left(2 \cdot 2^{-j_{n}}-2 \cdot 2^{-l_{n}}\right)^{N}-\left(4 \cdot 2^{-l_{n}}\right)^{N}
\end{aligned}
$$

Thus

$$
\frac{\mathcal{H}^{N}\left(\bigcup_{Q^{\prime} \in \mathcal{Q}(Q)} Q^{\prime}\right)}{\mathcal{H}^{N}(Q)}=\left(1-2^{j_{n}-l_{n}}\right)^{N}-\left(2 \cdot 2^{j_{n}-l_{n}}\right)^{N} .
$$

Note that

$$
\mathcal{H}^{N}(\mathcal{I})=\lim _{n \rightarrow \infty} \mathcal{H}^{N}\left(\cup_{Q^{\prime} \in \mathcal{Q}_{n}} Q^{\prime}\right)
$$

Further, for $n \geq 1$,

$$
\begin{aligned}
\mathcal{H}^{N}\left(\bigcup_{Q^{\prime} \in \mathcal{Q}_{n}} Q^{\prime}\right) & =\mathcal{H}^{N}\left(\bigcup_{Q \in \mathcal{Q}_{n-1}} \bigcup_{Q^{\prime} \in \mathcal{Q}(Q)} Q^{\prime}\right)=\sum_{Q \in \mathcal{Q}_{n-1}} \mathcal{H}^{N}\left(\bigcup_{Q^{\prime} \in \mathcal{Q}(Q)} Q^{\prime}\right) \\
& =\sum_{Q \in \mathcal{Q}_{n-1}} \frac{\mathcal{H}^{N}\left(\bigcup_{Q^{\prime} \in \mathcal{Q}(Q)} Q^{\prime}\right)}{\mathcal{H}^{N}(Q)} \cdot \mathcal{H}^{N}(Q) \\
& =\left(\left(1-2^{j_{n-1}-l_{n-1}}\right)^{N}-\left(2 \cdot 2^{j_{n-1}-l_{n-1}}\right)^{N}\right) \mathcal{H}^{N}\left(\underset{Q \in \mathcal{Q}_{n-1}}{\bigcup Q}\right) .
\end{aligned}
$$

Thus

$$
\mathcal{H}^{N}(\mathcal{I})=\mathcal{H}^{N}\left((-1,1)^{N}\right) \cdot \prod_{n=0}^{\infty}\left(\left(1-2^{j_{n}-l_{n}}\right)^{N}-\left(2 \cdot 2^{j_{n}-l_{n}}\right)^{N}\right)
$$

Inequality (23) and Lemma 6.2 now complete the proof.

Acknowledgments $\quad$ K. W. was supported by Academy of Finland Grant 128144, the Swiss National Science Foundation, European Research Council Project CG-DICE, and the European Science Council Project HCAA. T. Z. was supported by the Swiss National Science Foundation Grant PBBEP3_130157 and by the Academy of Finland Grant Number 251650. 


\section{References}

1. Burago, D., Burago, Y., Ivanov, I.: A course in metric geometry. Graduate Studies in Mathematics, vol. 33. American Mathematical Society, Providence (2001)

2. Balogh, Z.M., Csörnyei, M.: Scaled-oscillation and regularity. Proc. Am. Math. Soc. 134(9), 26672675 (2006) (electronic)

3. Balogh, Z.M., Rogovin, K., Zürcher, T.: The Stepanov differentiability theorem in metric measure spaces. J. Geom. Anal. 14, 405-422 (2004)

4. Bennett, C., Sharpley, R.: Interpolation of operators. Pure Appl. Math., vol. 129. Academic Press, Boston (1988)

5. Calderón, A.P.: On the differentiability of absolutely continuous functions. Rivista Mat. Univ. Parma 2, 203-213 (1951)

6. Cesari, L.: Sulle funzioni assolutamente continue in due variabili. Ann. Scuola Norm. Super. Pisa 2(10), 91-101 (1941)

7. Cheeger, J.: Differentiability of Lipschitz functions on metric measure spaces. Geom. Funct. Anal. 9(3), 428-517 (1999)

8. Cheeger, J., Kleiner, B.: On the differentiability of Lipschitz maps from metric measure spaces to Banach spaces. Nankai Tracts Math., vol. 11, pp. 129-152. World Sci. Publ, Hackensack (2006)

9. Cheeger, J., Kleiner, B.: Differentiability of Lipschitz maps from metric measure spaces to Banach spaces with the Radon-Nikodým property. Geom. Funct. Anal. 19(4), 1017-1028 (2009)

10. Cheeger, J., Kleiner, B., Naor, A.: Compression bounds for Lipschitz maps from the Heisenberg group to $L_{1}$. Acta Math. 207(2), 291-373 (2011)

11. David, G., Semmes, S.: Fractured Fractals and Broken Dreams: Self-Similar Geometry Through Metric and Measure. Oxford University Press, Oxford (1997)

12. Federer, H.: Geometric measure theory. Die Grundlehren der mathematischen Wissenschaften, Band 153. Springer-Verlag, New York (1969)

13. Gong, J.: The Lip-lip condition on metric measure spaces. arXiv:1208.2869.

14. Hanson, B.: Linear dilatation and differentiability of homeomorphisms of $\mathbb{R}^{n}$. Proc. Am. Math. Soc. 140(10), 3541-3547 (2012)

15. Heinonen, J.: Lectures on analysis on metric spaces. Universitext. Springer-Verlag, New York (2001)

16. Heinonen, J., Koskela, P.: Quasiconformal maps in metric spaces with controlled geometry. Acta Math. 181(1), 1-61 (1998)

17. Hajłasz, P., Koskela, P.: Sobolev met Poincaré. Mem. Am. Math. Soc 145(688), x+101 pp. (2000)

18. Heinonen, J., Koskela, P., Shanmugalingam, N., Tyson, J.T.: Sobolev classes of Banach space-valued functions and quasiconformal mappings. J. Anal. Math. 85, 87-139 (2001)

19. Jerison, D.: The Poincaré inequality for vector fields satisfying Hörmander's condition. Duke Math. J. 53(2), 503-523 (1986)

20. Johnson, W.B., Lindenstrauss, J., Schechtman, G.: Extensions of Lipschitz maps into Banach spaces. Israel J. Math. 54(2), 129-138 (1986)

21. Keith, S.: Modulus and the Poincaré inequality on metric measure spaces. Math. Z. 245(2), 255-292 (2003)

22. Keith, S.: A differentiable structure for metric measure spaces. Adv. Math. 183(2), 271-315 (2004)

23. Kauhanen, J., Koskela, P., Malý, J.: On functions with derivatives in a Lorentz space. Manuscripta Math. 100(1), 87-101 (1999)

24. Kleiner, B., MacKay, J.: Differentiable structures on metric measure spaces: a primer. arXiv:1108.1324v1.

25. Koskela, P., MacManus, P.: Quasiconformal mappings and Sobolev spaces. Stud Math. 131(1), 1-17 (1998)

26. Keith, S., Zhong, X.: The Poincaré inequality is an open ended condition. Ann. Math 167(2), 575-599 (2008)

27. Lee, J.R., Naor, A.: Extending Lipschitz functions via random metric partitions. Invent. Math. 160(1), 59-95 (2005)

28. Lang, U., Schlichenmaier, T.: Nagata dimension, quasisymmetric embeddings, and Lipschitz extensions. Int. Math. Res. Not. 58, 3625-3655 (2005)

29. Malý, J.: Absolutely continuous functions of several variables. J. Math. Anal. Appl. 231(2), 492-508 (1999) 
30. Malý, J.: Sufficient conditions for change of variables in integral. In Proceedings on Analysis and Geometry (Russian) (Novosibirsk Akademgorodok, 1999), pages 370-386. Izdat. Ross. Akad. Nauk Sib. Otd. Inst. Mat., Novosibirsk, 2000.

31. Malý, J., Swanson, D., Ziemer, W.P.: Fine behavior of functions whose gradients are in an Orlicz space. Stud. Math. 190(1), 33-71 (2009)

32. Pansu, P.: Métriques de Carnot-Carathéodory et quasiisométries des espaces symétriques de rang un. Ann. Math. 129(1), 1-60 (1989)

33. Rademacher, H.: Über partielle und totale Differenzierbarkeit von Funktionen mehrerer Variabeln und über die Transformation der Doppelintegrale. Math. Ann. 79(4), 340-359 (1919)

34. Ranjbar-Motlagh, A.: An embedding theorem for Sobolev type functions with gradients in a Lorentz space. Stud. Math. 191(1), 1-9 (2009)

35. Romanov, A.: Absolute continuity of the Sobolev type functions on metric spaces. Siberian. Math. J. 49(5), 911-918 (2008)

36. Schioppa, A.: On the relationship between derivations and measurable differentiable structures on metric measure spaces. Ann. Acad. Sci. Fenn. Math. 39, 275-304 (2014)

37. Shanmugalingam, N.: Newtonian spaces: an extension of Sobolev spaces to metric measure spaces. Rev. Mat. Iberoamericana. 16(2), 243-279 (2000)

38. Stepanoff, W.: Über totale Differenzierbarkeit. Math. Ann. 90, 318-320 (1923)

39. Stein, E.M.: Editor's note: The differentiability of functions in $R^{n}$. Ann. Math. 113(2), 383-385 (1981)

40. Wildrick, K., Zürcher, T.: Mappings with an upper gradient in a Lorentz space. http://www.math.jyu. fi/research/pspdf/382.pdf, 2009. Preprint 382

41. Wildrick, K., Zürcher, T.: Space filling with metric measure spaces. Math. Z. 270, 103-131 (2012). doi:10.1007/s00209-010-0787-1

42. Zürcher, T.: Local Lipschitz numbers and Sobolev spaces. Mich. Math. J. 55(3), 561-574 (2007) 